\title{
Comparisons of exact results for the virtual photon contribution to single hard bremsstrahlung in radiative return for $e^{+} e^{-}$annihilation
}

\author{
S. Jadach \\ CERN, Theory Division, CH-1211 Geneva 23, Switzerland and Institute of Nuclear Physics, ul. Radzikowskiego 152, Kraków, Poland \\ B. F. L. Ward and S. A. Yost \\ Department of Physics, Baylor University, One Bear Place \#97316, Waco, Texas 76798-7316, USA
}

(Received 21 February 2006; published 5 April 2006)

\begin{abstract}
We compare fully differential exact results for the virtual photon correction to single hard photon bremsstrahlung obtained using independent calculations, both for $e^{+} e^{-}$annihilation at high-energy colliders and for radiative return applications. The results are compared using Monte Carlo evaluations of the matrix elements as well as by direct analytical evaluation of certain critical limits. Special attention is given to the issues of numerical stability and the treatment of finite-mass corrections. It is found that agreement on the order of $10^{-5}$ or better is obtained over most of the range of hard photon energies, at CMS energies relevant to both high-energy collisions and radiative return experiments.
\end{abstract}

DOI: 10.1103/PhysRevD.73.073001

PACS numbers: 12.20.Ds

\section{INTRODUCTION}

The single hard bremsstrahlung process is critical in precision studies of standard model physics over a wide range of scales, from the $1 \mathrm{GeV}$ regime, where it is exploited in radiative return studies of intermediate-energy hadron physics, to the $100 \mathrm{GeV}$ regime and beyond, where it is used for radiative return studies of the $Z$ resonance. Construction of a future linear collider would push the energy range of interest toward the $\mathrm{TeV}$ regime. Precision studies of per mille level effects in any of these regimes requires exact calculations of all QED effects at order $\alpha^{2}$. For radiative return, this means that the virtual photon corrections to single hard photon radiation must be included.

Exact results for the virtual corrections to single hard bremsstrahlung have been studied by a number of groups [1-4]. Comparisons of these results are important to be certain of their correctness at a high level of precision. In Ref. [3], we have previously presented comparisons of the results in Refs. [1-3], and in general a very good agreement was found. However, it is clear from the comparisons in Ref. [3] that small differences appear at the level of the next-to-next-to-leading-log (NNLL), suggesting differences in the levels of "exactness" in the calculations.

These differences are largely due to how differential the results are, and how the mass corrections are included. Both Ref. [3] (JMWY) and Ref. [1] include mass corrections, but the former is fully differential, while the latter averages over the photon angle. The results of Ref. [2] are fully differential, but the mass corrections are neglected. These comparisons therefore are not a complete test of the results in Ref. [3] at the NNLL level.

Another calculation of the exact virtual corrections to single hard bremsstrahlung has appeared recently. An exact differential result including mass corrections needed for an accurate calculation of collinear emission is presented in
Ref. [4] (KR). This result was obtained for use in radiative return studies of hadronic final states [5-7]. The virtual correction without explicit mass terms [8] was obtained for use in tagged photon experiments, and this was extended to the small angle regime for untagged experiments with the inclusion of finite-mass corrections [4], which become significant in collinear emission, even in very high-energy collisions.

The results of KR have been implemented in the PHOKHARA MC [4,8-10], while the results of Ref. [3] have been implemented in the $\mathcal{K} \mathcal{K}$ MC [11]. In Ref. [12], an indirect comparison of the JMWY and KR results has been reported via comparison of the PHOKHARA and $\mathcal{K} \mathcal{K}$ MC's. Agreement at the per mille level was reported for selected observables. Further comparisons of these MC's were reported in Ref. [13], where the effect of including YFS (Yennie-Frautschi-Suura) exponentiation $[14]$ in the $\mathcal{K} \mathcal{K}$ MC versus the unexponentiated approach of PHOKHARA plays an important role in the differences found.

Presently, our interest is not in comparing the MC's but in comparing the order $\alpha^{2}$ matrix elements for hard photon emission used in each. A direct comparison of the matrix elements JMWY and KR was reported in Ref. [15], using a common MC evaluation of both expressions. It was found that the expressions agree to $1.5 \times 10^{-5}$ units of the Born cross section for a photon energy below $95 \%$ of the beam energy at $200 \mathrm{GeV}$ CMS energy.

This level of agreement was the result of careful analytical work to cancel large terms in the expressions of KR. The published matrix elements of KR and JMWY have very different forms, which is evident in part from the appearance of mass corrections proportional to $\left(p_{i} \cdot k\right)^{-2}$ and $\left(p_{i} \cdot k\right)^{-3}$ in the expressions of $\mathrm{KR}$, where $p_{i}$ is an incoming fermion momentum and $k$ is the emitted hard photon momentum. Such terms are absent in the JMWY 
expression, and we have verified that they cancel exactly in the KR expression as well, although this is not immediately obvious. This cancellation should be implemented analytically to obtain a stable MC comparison of the results. Such an analytical cancellation is presented here.

The functions used to construct the stable comparisons were introduced in Ref. [16]. A proof that the massless limits of the KR and JMWY expressions agree at next-toleading-log (NLL) order was sketched and the size of the difference in the mass corrections was calculated in Ref. [17]. MC results for a comparison of the matrix elements in the $1 \mathrm{GeV}$ radiative return regime were also obtained [18]. In this paper, we will present details of the stabilization procedure needed for very high precision comparisons, and present new MC comparisons which use these versions of the matrix elements, which have been improved since the publication of Refs. [15-18] for better behavior at lower CMS energies.

An important part of this comparison is an examination of the finite-mass corrections used in the virtual corrections of KR and JMWY. The electron mass terms containing denominators of the form $p_{i} \cdot k$ give contributions of the same order as some of the "massless" contributions upon integration. Therefore, the finite-mass corrections of both calculations are compared explicitly, and it is shown that both mass corrections are identical to NLL order. This supplements the result that the massless results agree to NLL order, showing that the complete virtual corrections agree up to NNLL terms. These NNLL terms are the subject of the numerical comparisons, both with and without mass terms.

We need to point out that both approaches to the exact mass corrections discussed here, that used by JMWY and that used by KR, should have the property that, upon integrating over the final phase space, the resulting integrated cross section should contain all finite terms that do not vanish for $m_{e}^{2} / s \rightarrow 0$. Both approaches use expansions therefore in the parameter $m_{e}^{2} / s$. The JMWY approach uses the expansion constructed by Ref. [19]. The KR approach uses the expansion of the respective leptonic tensor coefficients through terms of order $m_{e}^{4} s /\left(2 k \cdot p_{i}\right)^{3}$, where $k$ is the radiated real photon four momentum and $p_{i}$ is an incoming $e^{-}$or $e^{+}$four momentum. It is therefore important to know the relationship between these expansions both numerically and theoretically. We address this relationship matter in what follows.

The structure of this paper is as follows. In Section II, the matrix elements of JMWY and KR are displayed explicitly in a compatible format appropriate for comparisons, without the mass corrections in each case. Both forms of the matrix elements are shown in a form explicitly stable in collinear limits, which is suitable both for MC evaluation at very high precision, and for an analytical investigation of the collinear limits. A comparison of the collinear limits is presented in Section III, demonstrating that both matrix elements agree to NLL order when the explicit mass terms are omitted. Section IV examines the mass terms which are added in each case, and verifies that these both agree as well to NLL order, and to NNLL order in the soft limit. A numerical comparison of the matrix elements is presented in Section $\mathrm{V}$, both at $1 \mathrm{GeV}$ and $500 \mathrm{GeV}$ CMS energies. Finally, Section VI summarizes our conclusions, and the appendix presents details on the special functions introduced to stabilize the collinear limits.

\section{VIRTUAL CORRECTIONS TO INITIAL-STATE RADIATION}

The process we consider is initial-state radiation (ISR) in $e^{+} e^{-} \rightarrow \mu^{+} \mu^{-}$, where one real and one virtual photon are radiated on the electron-positron line. In Ref. [3], the matrix element was evaluated using helicity amplitude techniques of CALKUL [20], Xu et al. [21], and KleissStirling [22], together with algebraic simplification using FORM [23]. The mass corrections were then added following the methods in Ref. [19], after checking that the exact expression for the mass corrections differs from the result obtained by the latter methods by terms which vanish as $m_{e}^{2} / s \rightarrow 0$ in the integrated cross section, where $m_{e}$ is the electron mass and $s$ is the squared CMS energy.

In Ref. [4], the same ISR matrix element's interference with the Born process is calculated in terms of a leptonic tensor in such a way that all mass effects relevant in the collinear limits are obtained using an expansion in powers of $m_{e}^{2} / p_{i} \cdot k$. Thus, comparison of the two sets of results gives important information on the two different methods of calculation and on the two different treatments of the mass corrections.

The JMWY result used in these comparisons will be evaluated without $Z$ boson exchange, to match the calculation of KR. We denote the four momenta and helicity of the $e^{-}, e^{+}, f$, and $\bar{f}$ as $p_{j}$ and $\lambda_{j}, j=1, \ldots, 4$, respectively. The tree-level cross section for single hard ISR for QED alone may be written as

$$
\frac{d \sigma_{1}^{\mathrm{ISR}(0)}}{d \Omega d r_{1} d r_{2}}=\frac{1}{16(4 \pi)^{4}} \sum_{\lambda_{i}, \sigma}\left|\mathcal{M}_{1}^{\mathrm{ISR}(0)}\right|^{2}
$$

where the squared matrix element, summed over helicities, is

$$
\begin{aligned}
\sum_{\lambda_{i}, \sigma}\left|\mathcal{M}_{1}^{\mathrm{ISR}(0)}\right|^{2}= & \frac{16 e^{6}}{s^{2} s^{\prime} r_{1} r_{2}}\left\{\left(t_{1}^{2}+u_{1}^{2}\right)\left(1-\frac{2 m_{e}^{2} r_{1}}{s^{\prime} r_{2}}\right)\right. \\
& \left.+\left(t_{2}^{2}+u_{2}^{2}\right)\left(1-\frac{2 m_{e}^{2} r_{2}}{s^{\prime} r_{1}}\right)\right\},
\end{aligned}
$$

with $s=\left(p_{1}+p_{2}\right)^{2}, s^{\prime}=\left(p_{3}+p_{4}\right)^{2}, t_{1}=\left(p_{1}-p_{3}\right)^{2}$, $t_{2}=\left(p_{2}-p_{4}\right)^{2}, u_{1}=\left(p_{1}-p_{4}\right)^{2}, u_{2}=\left(p_{2}-p_{3}\right)^{2}, r_{i}=$ $2 p_{i} \cdot k / s$, and the solid angle $d \Omega$ refers to the direction of $p_{3}-p_{4}$ relative to $p_{1}-p_{2}$ in the CM frame of the out- 
going fermions. The explicit mass corrections in Eq. (2) are those obtained using the method of Ref. [19].

The same tree-level matrix element may be expressed in terms of the leptonic tensor $L^{\mu \nu}$ of Refs. [4,8]. In the same notation, the leading-order contribution to the leptonic tensor may be written (in the normalization of Ref. [8])

$$
\begin{aligned}
L_{0}^{\mu \nu}= & \frac{e^{4}}{s\left(s^{\prime}\right)^{2} r_{1} r_{2}}\left\{a_{00}^{(0)} s \eta^{\mu \nu}+a_{11}^{(0)} p_{1}^{\mu} p_{1}^{\nu}+a_{22}^{(0)} p_{2}^{\mu} p_{2}^{\nu}\right. \\
& \left.+a_{12}^{(0)}\left(p_{1}^{\mu} p_{2}^{\nu}+p_{2}^{\mu} p_{1}^{\nu}\right)\right\}
\end{aligned}
$$

with

$$
\begin{aligned}
& a_{00}^{(0)}=-\left(1-r_{1}\right)^{2}-\left(1-r_{2}\right)^{2}+\frac{2 m_{e}^{2} z v^{2}}{s r_{1} r_{2}}, \\
& a_{11}^{(0)}=-4 z+\frac{8 m_{e}^{2} r_{1}}{s r_{2}}, \\
& a_{22}^{(0)}=-4 z+\frac{8 m_{e}^{2} r_{2}}{s r_{1}}, \\
& a_{12}^{(0)}=\frac{-8 m_{e}^{2}}{s}
\end{aligned}
$$

where $z=s^{\prime} / s=1-v$ with $v=r_{1}+r_{2}$. This may be contracted with a final state tensor for a muon line,

$$
M^{\mu \nu}=e^{2}\left\{p_{3}^{\mu} p_{4}^{\nu}+p_{4}^{\mu} p_{3}^{\nu}-\left(p_{3} \cdot p_{4}\right) \eta^{\mu \nu}\right\},
$$

to obtain the same squared matrix element as in Eq. (2),

$$
\begin{aligned}
\sum_{\lambda_{i}, \sigma}\left|\mathcal{M}_{1}^{\mathrm{ISR}(0)}\right|^{2}= & 16 L_{\mu \nu}^{(0)} M^{\mu \nu} \\
= & \frac{8 e^{6}}{s\left(s^{\prime}\right)^{2} r_{1} r_{2}}\left\{-s s^{\prime}\left(2 a_{00}^{(0)}+a_{12}^{(0)}\right)+a_{11}^{(0)} t_{1} u_{1}\right. \\
& \left.+a_{22}^{(0)} t_{2} u_{2}+a_{12}^{(0)}\left(t_{1} t_{2}+u_{1} u_{2}\right)\right\}
\end{aligned}
$$

where the coefficient is chosen to match the normalization of Eq. (2), and explicit mass terms are kept only when enhanced by collinear factors. The mass terms in Eqs. (2) and (6) agree in the collinear limits where $r_{i}$ is of order $m_{e}^{2} / s$.

The initial-state virtual photon corrections to the cross section may be expressed as

$$
\frac{d \sigma_{1}^{\mathrm{ISR}(1)}}{d \Omega d r_{1} d r_{2}}=\frac{1}{16(4 \pi)^{4}} \sum_{\lambda_{i}, \sigma} 2 \operatorname{Re}\left[\left(\mathcal{M}_{1}^{\mathrm{ISR}(0)}\right)^{*} \mathcal{M}_{1}^{\mathrm{ISR}(1)}\right]
$$

where the matrix element for hard photon initial-state emission with one virtual photon may be expressed as

$$
\mathcal{M}_{1}^{\operatorname{ISR}(1)}=\frac{\alpha}{4 \pi} \mathcal{M}_{1}^{\operatorname{ISR}(0)}\left(f_{0}+f_{1} I_{1}+f_{2} I_{2}\right),
$$

where $f_{i}$ are scalar form factors and $I_{i}$ are spinor factors defined in Ref. [3]:

$$
\begin{aligned}
& I_{1}=\sigma \lambda_{3} s_{\lambda_{1}}\left(p_{1}, k\right) s_{-\lambda_{1}}\left(p_{2}, k\right) \frac{s_{\lambda_{3}}\left(p_{4}, p_{2}\right) s_{-\lambda_{3}}\left(p_{2}, p_{3}\right)-s_{\lambda_{3}}\left(p_{4}, p_{1}\right) s_{-\lambda_{3}}\left(p_{1}, p_{3}\right)}{s_{-\sigma}\left(p_{1}, p_{2}\right) s_{-\sigma}\left(p_{3}, p_{4}\right) s_{\sigma}^{2}\left(p_{21}, p_{34}\right)}, \\
& I_{2}=\lambda_{1} \lambda_{3} \frac{s_{\lambda_{1}}\left(p_{1}, k\right) s_{-\lambda_{1}}\left(p_{2}, k\right) s_{\lambda_{3}}\left(p_{4}, k\right) s_{-\lambda_{3}}\left(p_{3}, k\right)}{s_{-\sigma}\left(p_{1}, p_{2}\right) s_{-\sigma}\left(p_{3}, p_{4}\right) s_{\sigma}^{2}\left(\tilde{p}_{12}, p_{34}\right)},
\end{aligned}
$$

where $\lambda_{i}$ is the helicity of the fermion with momentum $p_{i}$ and $\sigma$ is the photon helicity. The spinor product is $s_{\lambda}(p, q)=$ $\bar{u}_{-\lambda}(p) u_{\lambda}(q)$, and

$$
\left(p_{i j}, \tilde{p}_{i j}\right)=\left\{\begin{array}{l}
\left(p_{i}, p_{j}\right) \\
\left(p_{j}, p_{i}\right)
\end{array} \quad \text { for } \sigma=\left\{\begin{array}{l}
\lambda_{i} \\
\lambda_{j}
\end{array} .\right.\right.
$$

The form factors $f_{i}$ are given (without mass corrections) by

$$
\begin{aligned}
f_{0}= & 4 \pi B_{\mathrm{YFS}}(s)+2(L-1-i \pi)+\frac{r_{2}}{1-r_{2}}+\frac{r_{2}\left(2+r_{1}\right)}{\left(1-r_{1}\right)\left(1-r_{2}\right)}\left\{\ln \left(\frac{r_{2}}{z}\right)+i \pi\right\}-\left\{3 v+\frac{2 r_{2}}{1-r_{2}}\right\} \mathrm{Lf}_{1}(-v) \\
& +\frac{v}{\left(1-r_{2}\right)} R_{1}\left(r_{1}, r_{2}\right)+r_{2} R_{1}\left(r_{2}, r_{1}\right), \\
f_{1}= & \frac{r_{1}-r_{2}}{2\left(1-r_{1}\right)\left(1-r_{2}\right)}+\frac{z}{\left(1-r_{1}\right)\left(1-r_{2}\right)}\left(r_{2}+\frac{1+z}{2\left(1-r_{1}\right)}\right)\left\{\ln \left(\frac{r_{2}}{z}\right)+i \pi\right\}+\frac{z}{1-r_{2}}\left\{\frac{1}{2} R_{1}\left(r_{1}, r_{2}\right)+r_{2} R_{2}\left(r_{1}, r_{2}\right)\right\} \\
& +\frac{v}{4}\left\{R_{1}\left(r_{1}, r_{2}\right) \delta_{\sigma, 1}+R_{1}\left(r_{2}, r_{1}\right) \delta_{\sigma,-1}\right\}, \\
f_{2}= & v+\frac{r_{1} r_{2}-v / 2}{\left(1-r_{1}\right)\left(1-r_{2}\right)}-2 v z \mathrm{Lf}_{3}(-v)+\frac{z}{\left(1-r_{1}\right)\left(1-r_{2}\right)}\left(2-r_{2}+\frac{r_{2}-r_{1}}{2\left(1-r_{1}\right)}\right)\left\{\ln \left(\frac{r_{2}}{z}\right)+i \pi\right\} \\
& +\frac{z}{1-r_{2}}\left\{\frac{1}{2} R_{1}\left(r_{1}, r_{2}\right)+\left(2-r_{2}\right) R_{2}\left(r_{1}, r_{2}\right)\right\}+\frac{r_{1}-r_{2}}{4}\left\{R_{1}\left(r_{1}, r_{2}\right) \delta_{\sigma, 1}+R_{1}\left(r_{2}, r_{1}\right) \delta_{\sigma,-1}\right\}
\end{aligned}
$$

for photon helicity $\sigma=\lambda_{1}$. When $\sigma=-\lambda_{1}, r_{1}$, and $r_{2}$ must be interchanged. Here, $L=\ln \left(s / m_{e}^{2}\right)$ is the "large logarithm" which may be used in a leading log expansion of the results. Thus, leading log (LL) refers to order $L^{2}$, 
NLL to order $L$, and NNLL to order $L^{0}=1$ in this expansion.

The infrared divergence is contained in the virtual YFS form factor defined by [14]

$$
4 \pi B_{\mathrm{YFS}}(s)=\left(2 \ln \frac{m_{\gamma}^{2}}{m_{e}^{2}}+1\right)(L-1-i \pi)-L^{2}-1+\frac{4 \pi^{2}}{3}+i \pi(L-1) .
$$

We also make use of functions

$$
\begin{aligned}
R_{1}(x, y)= & \operatorname{Lf}_{1}(-x)\left\{\ln \left(\frac{1-x}{y^{2}}\right)-2 \pi i\right\}+\frac{2(1-x-y)}{1-x} \operatorname{Sf}_{1}\left(\frac{y}{1-x}, \frac{x(1-x-y)}{1-x}\right), \\
R_{2}(x, y)= & \operatorname{Lf}_{1}(-x)-\frac{1}{2} \operatorname{Lf}_{1}^{2}(-x)+\frac{x}{x+y} \operatorname{Lf}_{2}(-x)+\frac{y}{(x+y)(1-x-y)} \operatorname{Lf}_{2}\left(\frac{y}{1-x-y}\right)+\operatorname{Lf}_{2}(-x)(\ln y+i \pi) \\
& +\left(\frac{1-x-y}{1-x}\right)^{2} \operatorname{Sf}_{2}\left(\frac{y}{1-x}, \frac{x(1-x-y)}{1-x}\right),
\end{aligned}
$$

with $\operatorname{Lf}_{n}(x), \mathrm{Sf}_{n}(x, y)$ defined in Eq. (A1a) of the appendix.

The functions $\mathrm{Lf}_{n}$ and $\mathrm{Sf}_{n}$ play an important role in canceling large factors in differences between logarithms or dilogarithms with slightly different arguments. Therefore, although the expressions for $f_{0}, f_{1}, f_{2}$ are analytically equivalent to those of Ref. [3], they are more stable when evaluated numerically. The functions $R_{1}$ and $R_{2}$ are related to the function $R$ used in Ref. [3] by

$R_{1}(x, y)=\frac{1}{x} R(x, y)$,

$R_{2}(x, y)=\frac{1}{x}\left\{\frac{1}{2} R_{1}(x, y)-\operatorname{Lf}_{1}(-x)+\ln \left(\frac{y}{1-x-y}\right)+i \pi\right\}$.

The functions $R_{i}$ are defined so that both are finite for $x, y \rightarrow 0$ with $x, y$ positive. This implies that $f_{1}$ and $f_{2}$ are finite for $r_{1}, r_{2} \rightarrow 0$. The spinor products $I_{1}$ and $I_{2}$ vanish in this limit, since $\left|s_{\lambda}\left(p_{i}, k\right)\right|^{2}=s r_{i}$. Therefore, the $f_{1}$ and $f_{2}$ terms are absent in the collinear limits.

The virtual corrections $a_{i j}^{(1)}$ to the coefficients $a_{i j}$ are calculated without explicit mass terms in Ref. [8]. The infrared divergence in that result is canceled by adding the contribution of an additional soft real photon with energy below a cutoff $v_{\min }$ as a fraction of the beam energy. To facilitate comparisons, the additional soft photon contribution will be removed, so that the pure virtual corrections are compared in each case. This requires decomposing the infrared-divergent term

$$
a_{i j}^{\mathrm{IR}}=a_{i j}^{(0)}\left\{2(L-1) \ln v_{\min }+\frac{3}{2}(L+\ln z)-2+\frac{\pi^{2}}{3}\right\}
$$

in Ref. [8] into a virtual contribution $a_{i j}^{(0)} F_{\mathrm{IR}}$ and a contribution due to an additional real soft photon. The correct decomposition is found to be

$$
a_{i j}^{\mathrm{IR}}=a_{i j}^{(0)}\left(F_{\mathrm{IR}}+2 \pi \tilde{B}_{\mathrm{YFS}}\left(s, v_{\min }\right)\right)
$$

with

$$
F_{\mathrm{IR}}=2 \pi \operatorname{Re} B_{\mathrm{YFS}}(s)+L-1+\frac{3}{2} \ln z
$$

and the real YFS soft photon form factor

$2 \pi \tilde{B}_{\mathrm{YFS}}\left(s, v_{\mathrm{min}}\right)=(L-1)\left\{\ln \frac{m_{e}^{2}}{m_{\gamma}^{2}}+2 \ln v_{\min }\right\}+\frac{1}{2} L^{2}-\frac{\pi^{2}}{3}$.

This can be verified by comparing the soft limit of the virtual correction to the known result, as is done at the end of this section.

We will use the functions $\mathrm{Lf}_{n}$ and $\mathrm{Sf}_{n}$ defined in the appendix to stabilize the results and clarify the collinear behavior. Then $a_{i j}=a_{i j}^{(0)}+\frac{\alpha}{\pi} a_{i j}^{(1)}$, with

$$
a_{i j}^{(1)}=\frac{\alpha}{\pi}\left(a_{i j}^{(0)} F_{\mathrm{IR}}+a_{i j}^{(1,0)}+a_{i j}^{(1, m)}\right),
$$

where the massless parts $a_{i j}^{(1,0)}$ of the non-IR-divergent part of the virtual corrections are given by 


$$
\begin{aligned}
a_{00}^{(1,0)}= & \frac{v z}{4}-\frac{r_{1} r_{2}}{2}+\frac{z}{2} \ln z+r_{1} r_{2} \operatorname{Lf}_{1}(-v)+\frac{r_{2}}{2}\left\{4-r_{2}-\frac{3(1+z)}{1-r_{1}}\right\} \ln \left(\frac{r_{2}}{z}\right)+\left\{r_{1}+\frac{r_{2}}{z}\left[1+\left(1-r_{2}\right)^{2}\right]\right\} \\
& \times\left\{z \ln \left(\frac{r_{2}}{z}\right) \operatorname{Lf}_{1}\left(-r_{1}\right)-\operatorname{Sf}_{1}\left(-\frac{r_{1}}{z},-\frac{r_{2}}{z}\right)\right\}+\left(r_{1} \leftrightarrow r_{2}\right), \\
a_{11}^{(1,0)}= & 3+z+\frac{(1+z)^{2}}{1-r_{1}}-6 \ln z-4 \operatorname{Lf}_{1}(-v)-4\left(1+r_{1}-r_{1} r_{2}\right)\left\{\operatorname{Lf}_{1}(-v)+\operatorname{Lf}_{2}(-v)\right\} \\
& \left.+2\left(\frac{v}{z}-z\left(1-r_{2}\right)\right) \operatorname{Lf}_{1}\left(\frac{r_{1} r_{2}}{z}\right)+2(1+z)\left\{\left(1-r_{2}\right) \operatorname{Lf}_{1}\left(-r_{2}\right)-\operatorname{Lf}_{1}\left(\frac{-r_{1}}{1-r_{2}}\right)-\frac{v}{z} \operatorname{Lf}_{1}\left(\frac{r_{2}}{z}\right)\right\}+2 \mathrm{Lf}_{1}\left(-r_{1}\right)\right\} \\
& -\frac{2}{z} \operatorname{Lf}_{1}\left(\frac{r_{1}}{z}\right)+2 r_{2} z \ln \left(\frac{r_{1}}{z}\right)\left\{\operatorname{Lf}_{1}\left(-r_{2}\right)+\operatorname{Lf}_{3}\left(-r_{2}\right)\right\}+r_{1} z \ln \left(\frac{r_{2}}{z}\right)\left\{r_{1}\left(\frac{1-r_{2}}{1-r_{1}}\right)^{2}+4 \operatorname{Lf}_{1}\left(-r_{1}\right)+2\left(1-r_{2}\right)^{2} \operatorname{Lf}_{3}\left(-r_{1}\right)\right\} \\
& -2 r_{2} \operatorname{Sf}_{1}\left(\frac{-r_{1}}{z}, \frac{-r_{2}}{z}\right)+\frac{2}{z} \operatorname{Sf}_{2}\left(\frac{-r_{1}}{z}, \frac{-r_{2}}{z}\right)-2\left(2+r_{1}-2 r_{2}\right) \operatorname{Sf}_{1}\left(\frac{-r_{2}}{z}, \frac{-r_{1}}{z}\right)+2 z \mathrm{Sf}_{2}\left(\frac{-r_{2}}{z}, \frac{-r_{1}}{z}\right), \\
a_{22}^{(1,0)}= & a_{11}^{(1,0)}\left(r_{1} \leftrightarrow r_{2}\right), \\
a_{12}^{(1,0)}= & \frac{z(1+z)}{2\left(1-r_{1}\right)\left(1-r_{2}\right)}+2 r_{1} r_{2}-\frac{v}{2}-z \operatorname{Lf}_{1}(-v)-2 z\left(1-r_{1} r_{2}\right) \operatorname{Lf}_{2}(-v)-z \operatorname{Lf}_{1}\left(\frac{r_{1} r_{2}}{z}\right) \\
& +2 z\left(r_{1} r_{2}+r_{2}-1\right)\left\{\frac{1}{1-r_{1}}+\operatorname{Lf}_{2}\left(-r_{1}\right)\right\} \ln \left(\frac{r_{2}}{z}\right)+\frac{(20 \mathrm{~b})}{\left(1-r_{2}\right)^{2}} \ln \left(\frac{r_{2}}{z}\right)-2\left(1-r_{1}\right) \operatorname{Sf}_{1}\left(\frac{-r_{1}}{z}, \frac{-r_{2}}{z}\right) \\
& +2 \operatorname{Sf}_{2}\left(\frac{-r_{1}}{z}, \frac{-r_{2}}{z}\right)+\left(r_{1} \leftrightarrow r_{2}\right) .
\end{aligned}
$$

These expressions are exactly equal to those of Ref. [8], but written in terms of the functions $\mathrm{Lf}_{n}$ and $\mathrm{Sf}_{n}$.

A comparison of the collinear limits of the two matrix elements will be presented in the following section. A numerical study comparing these two forms of the virtual photon correction to hard bremsstrahlung will be presented in Section $\mathrm{V}$, together with a comparison of the mass corrections added to obtain the complete matrix elements in each case.

\section{NLL COMPARISON OF THE MATRIX ELEMENTS}

In this section, we will verify that the expressions of Refs. [3,8] agree analytically to NLL order, meaning that they agree through order $L$ when integrated over $r_{i}$. For this purpose, we may examine both expressions in the limit of collinear emission, which is sufficient to determine the NLL result. We will consider the particular limit where $r_{1}$ becomes small, so that the photon is collinear with the incoming electron.

In the collinear limits, the virtual correction to the squared matrix element is proportional to the tree-level result,

$$
\sum_{\lambda_{i}, \sigma} 2 \operatorname{Re}\left(\mathcal{M}_{1}^{\mathrm{ISR}(0)^{*}} \mathcal{M}_{1}^{\mathrm{ISR}(1)}\right)=\frac{\alpha}{2 \pi} \operatorname{Re}\left\langle f_{0}^{\mathrm{NLL}}\right\rangle \sum_{\lambda_{i}, \sigma}\left|\mathcal{M}_{1}^{\mathrm{ISR}(0)}\right|^{2},
$$

where $\left\langle f_{0}^{\mathrm{NLL}}\right\rangle$ is the average of $f_{0}$ over helicities, evaluated for small $r_{1}$ or $r_{2}$ small, [3]

$$
\begin{aligned}
\operatorname{Re}\left\langle f_{0}^{\mathrm{NLL}}\right\rangle= & 2 \pi \operatorname{Re} B_{\mathrm{YFS}}(s)+L-1+3 \ln \left(1-r_{1}\right) \\
& +2 \ln r_{1} \ln \left(1-r_{2}\right)-\ln ^{2}\left(1-r_{1}\right)+2 \operatorname{Sp}\left(r_{1}\right) \\
& +\frac{r_{1}\left(1-r_{1}\right)}{1+\left(1-r_{1}\right)^{2}}+\left(r_{1} \rightarrow r_{2}\right)
\end{aligned}
$$

Since the $f_{1}, f_{2}$ terms in Eq. (8) both vanish in collinear limits, they do not contribute to NLL order. In the limit when $r_{1} \rightarrow 0$, the NLL expression may be written in the form

$$
\begin{aligned}
\operatorname{Re}\left\langle f_{0}^{\mathrm{NLL}}\left(r_{1} \rightarrow 0\right)\right\rangle= & F_{\mathrm{IR}}+2 \ln z \ln r_{1}-\ln ^{2} z+2 \operatorname{Sp}(v) \\
& +\frac{v z}{1+z^{2}}
\end{aligned}
$$

The result of Ref. [8] can be compared to this expression using Eq. (6) with $a_{i j}^{(0)}$ replaced by $a_{i j}^{(1)}$ in the collinear limit. The virtual correction to the squared matrix element may be written

$$
\begin{aligned}
\sum_{\lambda_{i}, \sigma} 2 \operatorname{Re}\left(\mathcal{M}_{1}^{\mathrm{ISR}(0)^{*}} \mathcal{M}_{1}^{\mathrm{ISR}(1)}\right)= & 16 L_{\mu \nu}^{(1)} M^{\mu \nu}=\frac{\alpha}{2 \pi}\left\{2 F_{\mathrm{IR}} \sum_{\lambda_{i}, \sigma}\left|\mathcal{M}_{1}^{\mathrm{ISR}(0)}\right|^{2}+\frac{8 e^{6}}{s\left(s^{\prime}\right)^{2} r_{1} r_{2}}\left[-s s^{\prime}\left(2 a_{00}^{(1,0)}+a_{12}^{(1,0)}\right)+a_{11}^{(1,0)} t_{1} u_{1}\right.\right. \\
& \left.\left.+a_{22}^{(1,0)} t_{2} u_{2}+a_{12}^{(1,0)}\left(t_{1} t_{2}+u_{1} u_{2}\right)\right]\right\} .
\end{aligned}
$$


In the collinear limit, this expression should again be proportional to the tree-level result, Eq. (2) without the mass terms. When $r_{1}$ is small, $t_{2}=z t_{1}+\mathcal{O}\left(r_{1}\right)$ and $u_{2}=z u_{1}+\mathcal{O}\left(r_{1}\right)$, allowing Eq. (24) to be written as

$$
\sum_{\lambda_{i}, \sigma} 2 \operatorname{Re}\left(\mathcal{M}_{1}^{\mathrm{ISR}(0)^{*}} \mathcal{M}_{1}^{\mathrm{ISR}(1)}\right)=\frac{\alpha}{2 \pi}\left\{f^{\prime} \sum_{\lambda_{i}, \sigma}\left|\mathcal{M}_{1}^{\mathrm{ISR}(0)}\right|^{2}+\frac{4 e^{6} \Delta}{s z r_{1} r_{2}}\right\}
$$

with

$$
f^{\prime}=2 F_{\mathrm{IR}}+\frac{1}{a_{00}^{(0)}}\left(a_{00}^{(1,0)}+\frac{\Delta}{4}\right), \quad \Delta=\frac{a_{11}^{(1,0)}}{z}+z a_{22}^{(1,0)}-2 a_{12}^{(1,0)}-4 a_{00}^{(1,0)},
$$

where $a_{00}^{(0)}=-\left(1+z^{2}\right)$ is the massless limit of the tree-level coefficient. When $r_{2}$ is small, the same form is obtained with $a_{11}$ and $a_{22}$ interchanged.

We may check that $\Delta=0$ and $f^{\prime}=\operatorname{Re} f_{0}$ in the collinear limits. Evaluating the coefficient functions in the limit when $k$ is collinear with $p_{1}$ gives

$$
\begin{aligned}
a_{00}^{(1,0)}\left(r_{1} \rightarrow 0\right)= & -\frac{v z}{2}+\left(1+z^{2}\right)\left\{\frac{1}{2} \ln ^{2} z-\ln z \ln r_{1}-\operatorname{Sp}(v)\right\}, \\
a_{11}^{(1,0)}\left(r_{1} \rightarrow 0\right)= & 3+2 z-2 \ln r_{1}\left\{z \ln z+\operatorname{Lf}_{1}(-v)+\operatorname{Lf}_{2}(-v)-\frac{1}{2}(1+v)\right\}+\ln z\left\{\operatorname{Lf}_{1}(-v)+2 \operatorname{Lf}_{2}(-v)-8\right\} \\
& +z \ln ^{2} z-2 z \operatorname{Sp}(v)-11 \operatorname{Lf}_{1}(-v)-\operatorname{Lf}_{1}^{2}(-v)-5 \operatorname{Lf}_{2}(-v)+2 \operatorname{Sf}_{1}(0, v)-2 \operatorname{Sf}_{2}(0, v), \\
a_{22}^{(1,0)}\left(r_{1} \rightarrow 0\right)= & \frac{1}{z}+2 z+\ln r_{1}\left\{\frac{1}{z}-4 z \ln z-2 \operatorname{Lf}_{1}(-v)-2 \operatorname{Lf}_{2}(-v)\right\}+\ln z\left\{\operatorname{Lf}_{1}(-v)+2 \operatorname{Lf}_{2}(-v)-\frac{3}{z}-4\right\} \\
& +2 z \ln ^{2} z-\operatorname{Lf}_{1}(-v)\left(\frac{2}{z}+7\right)-4 z \operatorname{Sp}(v)-\operatorname{Lf}_{1}^{2}(-v)-5 \operatorname{Lf}_{2}(-v)+2 \operatorname{Sf}_{1}(0, v)-2 \operatorname{Sf}_{2}(0, v), \\
a_{12}^{(1,0)}\left(r_{1} \rightarrow 0\right)= & 3+z+\ln r_{1}\left\{1-2 \operatorname{Lf}_{1}(-v)-2 \operatorname{Lf}_{2}(-v)\right\}+\ln z\left\{\operatorname{Lf}_{1}(-v)+2 \operatorname{Lf}_{2}(-v)-6-2 z\right\}-10 \operatorname{Lf}_{1}(-v) \\
& -\operatorname{Lf}_{1}^{2}(-v)-5 \operatorname{Lf}_{2}(-v)+2 \operatorname{Sf}_{1}(0, v)-2 \operatorname{Sf}_{2}(0, v) .
\end{aligned}
$$

Combining these relations confirms that $\Delta=0$ and $f^{\prime}=$ $\operatorname{Re} f_{0}$, so the results have the same collinear limits without explicit mass terms. This implies that the results without mass terms agree to NLL order.

Since the collinear limits agree, the soft limits also agree. Specifically, for $v \rightarrow 0$,

$$
a_{00}^{(1,0)}=0, \quad a_{11}^{(1,0)}=a_{22}^{(1,0)}=a_{12}^{(1,0)}=-3
$$

and $f^{\prime}=\operatorname{Re} f_{0}=4 \pi \operatorname{Re} B_{\mathrm{YFS}}(s)+2(L-1)$. This verifies that the virtual part of the infrared contribution was identified correctly in Eq. (16).

\section{COMPARISON OF MASS CORRECTIONS}

Explicit mass corrections are added in the JMWY result via the prescription of Ref. [19] and Sec. 3 of Ref. [24], leading to a correction which may be expressed as

$$
\begin{aligned}
\sum_{\lambda_{i}, \sigma} 2 \operatorname{Re}\left(\mathcal{M}_{1}^{\mathrm{ISR}(0)^{*}} \mathcal{M}_{1}^{\mathrm{ISR}(0)}\right)_{m}= & -\frac{m_{e}^{2}}{s^{\prime}} \frac{\alpha}{\pi} f_{\mathrm{NR}}\left(s^{\prime}\right) \frac{16 e^{6}}{s^{\prime}} \\
& \times\left\{\frac{t_{2}^{2}+u_{2}^{2}}{s^{2} r_{1}^{2}}+\frac{t_{1}^{2}+u_{1}^{2}}{s^{2} r_{2}^{2}}\right\},
\end{aligned}
$$

where the nonradiative virtual correction factor is

$$
f_{\mathrm{NR}}\left(s^{\prime}\right)=4 \pi B_{\mathrm{YFS}}\left(s^{\prime}\right)+2 \ln \left(\frac{s^{\prime}}{m_{e}^{2}}\right)-2
$$

with

$$
\begin{aligned}
4 \pi B_{\mathrm{YFS}}\left(s^{\prime}\right)= & 4 \pi B_{\mathrm{YFS}}(s)+\ln z\left(2 \ln \frac{m_{\gamma}^{2}}{m_{e}^{2}}-2 L+i \pi\right) \\
& -\ln ^{2} z .
\end{aligned}
$$

The first term in brackets in Eq. (29) is due to $e^{+}$line emission, and the second is due to $e^{-}$line emission.

This mass prescription is designed to produce the essential mass corrections in the collinear limits when the photon is emitted along the electron or positron line. In these limits, $r_{i}=m_{e}^{2} v / s$, where $i$ labels the momentum of the collinear incoming fermion line. The mass corrections of Ref. [4] may be compared analytically in this limit.

The explicit mass correction of KR is given by

$$
\sum_{\lambda_{i}, \sigma} 2 \operatorname{Re}\left(\mathcal{M}_{1}^{\mathrm{ISR}(0)^{*}} \mathcal{M}_{1}^{\mathrm{ISR}(0)}\right)_{m}=16 L_{\mu \nu}^{(1, m)} M^{\mu \nu},
$$

where only the mass corrections to the virtual part of the leptonic tensor are included, so that it is calculated using the coefficient functions 


$$
a_{i j}^{(m)}=\frac{\alpha}{\pi}\left(a_{i j}^{(0, m)} F_{\mathrm{IR}}+a_{i j}^{(1, m)}\right)
$$

with $F_{\mathrm{IR}}$ as in Eq. (17) and $a_{i j}^{(0, m)}$ given by the explicit mass terms in Eq. (4). The functions $a_{i j}^{(1, m)}$ are given in Ref. [4], and may be written in terms of $\rho_{i}=s r_{i} / m_{e}^{2}$ and the stabilized functions $\mathrm{Lf}_{n}$ and $\mathrm{Sf}_{n}$ as

$$
\begin{aligned}
a_{00}^{(1, m)}= & \frac{z r_{2}}{\rho_{1}}\left\{-\ln z\left(2 L-4 \ln \rho_{1}+\ln z\right)+4 \operatorname{Sp}(v)\right\}-z r_{2} \operatorname{Sf}_{1}\left(1,-\rho_{1}\right)-\frac{1}{2} z r_{2} N_{1}\left(\rho_{1}, \frac{1}{z}-4\right)+v r_{2} N_{3}\left(\rho_{1}\right) \\
& +\left(r_{1}, \rho_{1} \leftrightarrow r_{2}, \rho_{2}\right), \\
a_{11}^{(1, m)}= & \frac{4 r_{1}}{\rho_{2}}\left\{-\ln z\left(2 L-4 \ln \rho_{2}+\ln z\right)+4 \operatorname{Sp}(v)\right\}-4 r_{1}\left(\frac{z}{v}+2\right) \operatorname{Sf}_{1}\left(1,-\rho_{1}\right) \\
& +\frac{r_{1}}{v}\left\{4 z N_{3}\left(\rho_{2}\right)+\left(2 z-\frac{1}{z}\right) N_{1}\left(\rho_{2}, \frac{2(1-2 z)^{2}}{1-2 z^{2}}\right)+6 N_{2}\left(\rho_{2}\right)+2 N_{4}\left(\rho_{2}\right)\right\}+\frac{z r_{2}}{v}\left\{4 N_{3}\left(\rho_{1}\right)-N_{1}\left(\rho_{1}, 0\right)\right. \\
& \left.+2 z\left(N_{2}\left(\rho_{1}\right)+N_{4}\left(\rho_{1}\right)\right)\right\}, \\
a_{22}^{(1, m)}= & a_{11}^{(1, m)}\left(r_{1}, \rho_{1} \leftrightarrow r_{2}, \rho_{2}\right), \\
a_{12}^{(1, m)}= & \frac{z r_{2}}{v}\left\{4 N_{3}\left(\rho_{1}\right)+4 N_{2}\left(\rho_{1}\right)+2 N_{4}\left(\rho_{1}\right)+2 \operatorname{Sf}_{1}\left(1,-\rho_{1}\right)\right\}-r_{2} N_{1}\left(\rho_{1}, \frac{z}{v}\right)+\left(r_{1}, \rho_{1} \leftrightarrow r_{2}, \rho_{2}\right),
\end{aligned}
$$

where the functions $N_{i}$ are defined by

$$
\begin{aligned}
N_{1}(\rho, w)= & \operatorname{Lf}_{2}(\rho-1)-w \operatorname{Lf}_{2}(\rho-1), \\
N_{2}(\rho)= & \operatorname{Sf}_{1}(z, v-\rho)+\operatorname{Lf}_{1}(-v) \operatorname{Lf}_{1}\left(\frac{\rho}{v}-1\right) \\
= & \operatorname{Sf}_{1}(v, \rho-v)-\frac{1}{z} \ln \rho \operatorname{Lf}_{1}\left(\frac{v-\rho}{z}\right) \quad(\rho<1), \\
N_{3}(\rho)= & \frac{1}{\rho}\left(1-\ln \rho-\operatorname{Sf}_{1}(1,-\rho)\right) \\
= & -\ln \rho \operatorname{Lf}_{2}(-\rho)-\operatorname{Sf}_{2}(0, \rho) \quad(\rho<1) \\
N_{4}(\rho)= & \operatorname{Sf}_{2}(z, v-\rho)-\frac{1}{v} \operatorname{Lf}_{1}(-v) \operatorname{Lf}_{2}\left(\frac{\rho}{v}-1\right) \\
& -\frac{1}{v z} \operatorname{Lf}_{1}\left(\frac{\rho}{v}-1\right) \\
= & -\operatorname{Sf}_{2}(v, \rho-v)-\frac{1}{z^{2}} \ln \rho \operatorname{Lf}_{2}\left(\frac{v-\rho}{z}\right) \quad(\rho<1)
\end{aligned}
$$

All of the expressions $N_{i}$ are finite in the collinear limit $\rho \rightarrow v$ :

$$
\begin{aligned}
N_{1}(v, w) & =\frac{1}{z}-\operatorname{Lf}_{1}(-z)\left(\frac{1}{z}+w\right), \\
N_{2}(v) & =\operatorname{Lf}_{1}(-v)+\operatorname{Lf}_{1}(-z), \\
N_{3}(v) & =-\operatorname{Sf}_{2}(0, v)-\ln v \operatorname{Lf}_{2}(-v), \\
N_{4}(v) & =-\frac{1}{2}\left\{\operatorname{Lf}_{2}(-v)+\frac{1}{z}\left(1+\operatorname{Lf}_{1}(-z)\right)\right\} .
\end{aligned}
$$

Also, $\operatorname{Sf}_{1}(1,-\rho) \rightarrow \operatorname{Sf}_{1}(0, v)-\ln v \operatorname{Lf}_{1}(-v)$ in the collinear limit. All of the functions $N_{i}$ vanish in the limit $\rho \rightarrow \infty$, where masses are completely neglected.

In the collinear limit $\rho_{1} \rightarrow v$, corresponding to $e^{+}$line emission, the total mass correction functions $a_{i j}^{(m)}$ (including the infrared part) simplify to

$$
\begin{aligned}
a_{00}^{(m)}= & \frac{m_{e}^{2} v}{s r_{1}}\left\{2 z F_{\mathrm{IR}}+(3 z-1)(\ln v \ln z+\operatorname{Sp}(v))-2 z L \ln z-\frac{v^{2}}{z} \ln v-z \ln ^{2} z+\frac{v}{2}\right\} \\
a_{11}^{(m)}= & -\frac{m_{e}^{2} v}{s r_{1}}\left\{\ln v\left(\frac{v}{z}+2 z+4 z \mathrm{Lf}_{2}(-v)\right)+1+z-2 z^{2} \operatorname{Lf}_{1}(-v)+z^{2} \operatorname{Lf}_{2}(-v)+4 z \operatorname{Sf}_{2}(0, v)\right\} \\
a_{22}^{(m)}= & \frac{m_{e}^{2} v}{s r_{1}}\left\{8 F_{\mathrm{IR}}-8 L \ln z-\frac{1}{z^{3}}\left(1+z-4 z^{2}-8 z^{3}\right) \ln v+8 \ln v \ln z+8 \operatorname{Sp}(v)+4 z \ln v\left(\operatorname{Lf}_{1}(-v)-\operatorname{Lf}_{2}(-v)\right)\right. \\
& \left.-4 \ln ^{2} z+6 \operatorname{Lf}_{1}(-v)-\operatorname{Lf}_{2}(-v)-4 z\left(\operatorname{Sf}_{1}(0, v)+\operatorname{Sf}_{2}(0, v)\right)-\frac{1}{z^{2}}\left(1+z-2 z^{2}\right)\right\} \\
a_{12}^{(m)}= & \frac{m_{e}^{2} v}{s r_{1}}\left\{2 z \ln v\left(\operatorname{Lf}_{1}(-v)-2 \operatorname{Lf}_{2}(-v)\right)-\left(4+\frac{v^{2}}{z^{2}}\right) \ln v+4 z \operatorname{Lf}_{1}(-v)-z \operatorname{Lf}_{2}(-v)-2 z \operatorname{Sf}_{1}(0, v)-4 z \operatorname{Sf}_{2}(0, v)-\frac{1}{z}\right\}
\end{aligned}
$$


Note that there are no terms proportional to $\mathrm{m}^{4} / \mathrm{r}_{1}^{2}$, so that all such factors in Ref. [4] cancel when the terms are combined into the stabilized functions used here.

To verify that this result agrees with the JMWY result to NLL order, it is necessary to check all terms with explicit logarithms $L$ in the collinear limit. In the $e^{+}$line emission case, where $k$ is collinear with $p_{1}$,

$$
\begin{aligned}
\sum_{\lambda_{i}, \sigma} 2 \operatorname{Re}\left(\mathcal{M}_{1}^{\operatorname{ISR}(0)^{*}} \mathcal{M}_{1}^{\operatorname{ISR}(0)}\right)_{m}= & \frac{-4 e^{6}}{s^{2}\left(s^{\prime}\right)^{2} r_{1} r_{2}}\left\{\frac{4}{z}\left(t_{2}^{2}+u_{2}^{2}\right) f_{m}^{\prime}\right. \\
& \left.-z s^{2} \Delta_{m}\right\}
\end{aligned}
$$

with

$$
\begin{aligned}
f_{m}^{\prime} & =\frac{a_{11}^{(m)}}{4 z}+\frac{z a_{22}^{(m)}}{4}-\frac{a_{12}^{(m)}}{2}=2 F_{I R}-2 L \ln z+\mathcal{O}\left(L^{0}\right) \\
& =2 L\left(\ln \frac{m_{\gamma}^{2}}{m_{e}^{2}}+1-\ln z\right)-L^{2}+\mathcal{O}\left(L^{0}\right)
\end{aligned}
$$

and

$$
\Delta_{m}=\frac{a_{11}^{(m)}}{z}+z a_{22}^{(m)}-4 a_{00}^{(m)}=\mathcal{O}\left(L^{0}\right)
$$

Therefore,

$$
\begin{aligned}
\sum_{\lambda_{i}, \sigma} 2 \operatorname{Re}\left(\mathcal{M}_{1}^{\mathrm{ISR}(0)^{*}} \mathcal{M}_{1}^{\mathrm{ISR}(0)}\right)_{m}= & -\frac{m_{e}^{2}}{s^{\prime}} \frac{\alpha}{\pi} \frac{16 e^{6}}{s^{\prime}}\left(\frac{t_{2}^{2}+u_{2}^{2}}{s^{2} r_{1}^{2}}\right) \\
& \times\left\{2 L\left(\ln \frac{m_{\gamma}^{2}}{m_{e}^{2}}+1-\ln z\right)\right. \\
& \left.-L^{2}\right\}
\end{aligned}
$$

to NLL order. This agrees with the $e^{+}$line emission part of Eq. (29) to the same order, since

$$
f_{\mathrm{NR}}\left(s^{\prime}\right)=2 L \ln \frac{m_{\gamma}^{2}}{m_{e}^{2}}-L^{2}+2 L(1-\ln z)+\mathcal{O}\left(L^{0}\right) .
$$

Therefore, both expressions for the explicit mass terms agree analytically through NLL order.

We can also check that the mass terms agree exactly in the soft collinear limit. Taking $v \rightarrow 0$ in the collinear results for the coefficients $a_{i j}^{(m)}$ gives

$$
\begin{gathered}
a_{00}^{(m)}=2 F_{\mathrm{IR}}, \quad a_{11}^{(m)}=-1 / 2, \\
a_{22}^{(m)}=8 F_{\mathrm{IR}}+3 / 2, \quad a_{12}^{(m)}=1 / 2,
\end{gathered}
$$

which gives to Eq. (38) with $f_{m}^{\prime}=2 F_{\mathrm{IR}}$ and $\Delta_{m}=0$. In the soft limit, $f_{\mathrm{NR}}=F_{\mathrm{IR}}$, so the mass corrections are identical in the soft collinear limit.

\section{MONTE CARLO COMPARISONS}

Since both of the results compared are fully differential, they are well suited to MC implementation. The JMWY results were developed for the $\mathcal{K} \mathcal{K}$ MC described in Ref. [11], which is for high-energy fermion pair production. The results from Ref. [4] are implemented in the PHOKHARA MC developed for radiative return applications at $\Phi$ and $B$ factories. Structurally, these MC's are considerably different, with $\mathcal{K} \mathcal{K}$ MC implementing a YFS-exponentiated algorithm, and PHOKHARA unexponentiated. Some comparisons of the MC's have been reported $[12,13]$. Here, we will not compare the MC's, since that would test not just the comparison of the matrix elements, but also be affected by the presence or absence of exponentiation. Instead, we will implement both virtual photon corrections in the same MC, with the sole purpose of comparing the two matrix elements.

Since the results of JMWY were developed for use in the YFS-based Monte Carlo (MC) program $\mathcal{K} \mathcal{K}$ MC [11], we will look at how the results of $\mathcal{K} \mathcal{K}$ MC would change if the virtual correction of KR are substituted for those of JMWY in the same program. We calculate the YFS residuals $\bar{\beta}_{1}^{(i)}$ as defined in Refs. [11,15]. The residuals of interest here are for single photon emission, $\beta_{1}^{(i)}$ for $i=$ 1, 2 which were compared previously for several different virtual photon corrections in Refs. [15-18]. The superscript ( $i$ ) denotes the power of $\alpha$ relative to the born level, $\beta_{0}^{(0)}=d \sigma_{\text {Born }} / d \Omega$. Thus, $i=1$ corresponds to tree-level hard photon emission, and $i=2$ corresponds to the virtual corrections. The residuals are integrated up to a cutoff $v_{\max }$ on the photon energy fraction to produce cross sections denoted $\sigma_{\beta_{1}}^{(i)}$.

Results for comparisons of these YFS residuals were previously reported in Refs. [3,15-18] for the virtual photon corrections of Refs. [1,2] at a CMS energy of $200 \mathrm{GeV}$. Some results were also reported in Ref. [15] for $500 \mathrm{GeV}$. It was found in Ref. [15] that at $200 \mathrm{GeV}$, all of the fully differential results (excluding Ref. [1], which is not fully differential) agreed to within $5 \times 10^{-5}$ units of the Born cross section across the full range of $v$ investigated (up to $v_{\max }=0.9999$ ). Agreement to within $2 \times 10^{-4}$ units of the Born cross section was found at $500 \mathrm{GeV}$.

Here, we will restrict our comparisons to the two fully differential results including mass corrections, JMWY and $\mathrm{KR}$, discussed in the present paper. In addition to a comparison of the full results, we will compare the massless limits in which explicit mass terms in the virtual photon correction are omitted in both expressions. This will be useful in judging the size of the contribution of the mass corrections alone to the difference in the cross sections. Comparisons are made at CMS energies of $1 \mathrm{GeV}$ and $500 \mathrm{GeV}$. The $1 \mathrm{GeV}$ scale was chosen as representative of some of the radiative return experiments for which PHOKHARA was designed, while the $500 \mathrm{GeV}$ scale anticipates applications at a future linear collider (ILC). The $1 \mathrm{GeV}$ comparisons are essentially new, since the only previously reported comparisons at low energy [18] were made before the matrix elements were completely imple- 


\section{(a) $1 \mathrm{GeV}$}

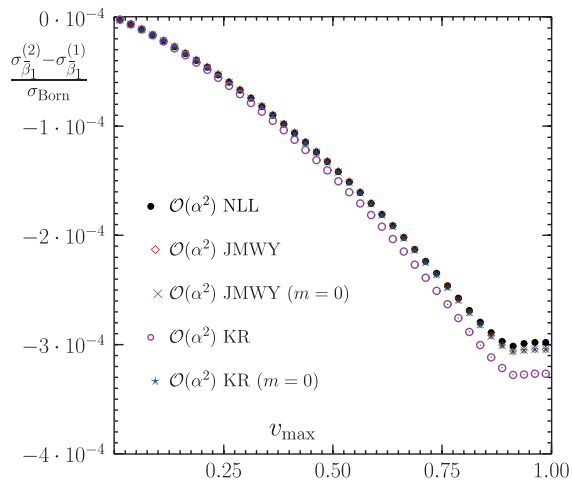

(b) $500 \mathrm{GeV}$

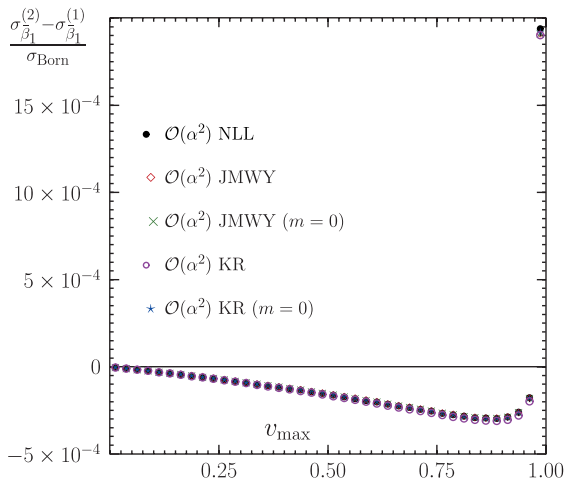

FIG. 1 (color online). The virtual photon contribution to the $\beta_{1}$ residual for muon pair production, integrated up to a cut $v_{\text {max }}$. Case (a) is for a CMS energy of $1 \mathrm{GeV}$, and case (b) is for a CMS energy of $500 \mathrm{GeV}$.

mented in their final form: it was found that some changes were needed to render the results sufficiently stable numerically when calculated for lower energy scales. The expressions shown in the previous sections are adequate for these calculations.

Figure 1 shows the virtual correction $\sigma_{\beta_{1}}^{(2)}-\sigma_{\beta_{1}}^{(1)}$, which is the pure $\mathcal{O}\left(\alpha^{2}\right)$ contribution to the single hard photon cross section. The results are obtained from $10^{8}$ events created using the YFS3ff generator, which is the EEX3 option in the $\mathcal{K} \mathcal{K}$ MC. The exact expressions are compared to a NLL approximation obtained from the expression Eq. (22). All cross sections in these comparisons are normalized by dividing by the radiationless Born cross section $\sigma_{\text {Born }}$ for $e^{+} e^{-} \rightarrow \mu^{+} \mu^{-}$. It is seen that at both $1 \mathrm{GeV}$ and $500 \mathrm{GeV}$, the agreement is very close between all the results compared.

To see the differences between the various matrix elements, it is necessary to look at the NNLL contribution, since we know from the analytical results in the previous sections that these expressions agree to NLL order. Figure 2 examines the differences between the JMWY and KR results and the NLL contribution of Eq. (22) at $1 \mathrm{GeV}$. This is a constant contribution (NNLL) to the cross

(a) integrated

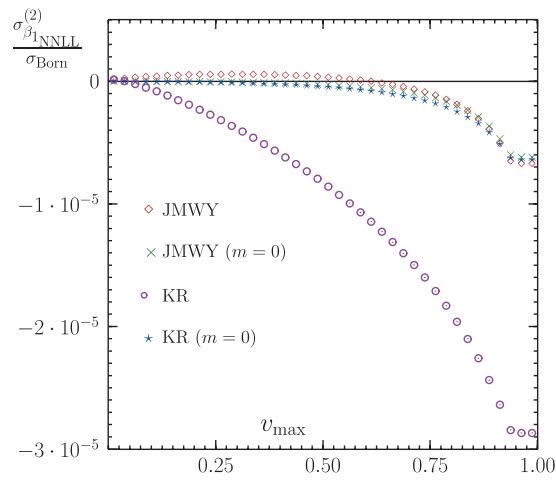

section at $\mathcal{O}\left(\alpha^{2}\right)$ with no factors of the big logarithm $L=$ $\ln \left(s / m_{e}^{2}\right)$. In FIG. 2(a), the difference between the integrated YFS residuals is shown, while in Fig. 2(b), the differences $d \sigma_{\bar{\beta}_{1}} / d v$ are plotted. In other words, Fig. 2(b) is the derivative of the plot in Fig. 2(a), showing the difference between the virtual corrections at each photon energy $v$. It is found that the complete results of JMWY and KR differ by at most $2.2 \times 10^{-5}$ units of the Born cross section when integrated over the full range of $v$.

The differential plot shows that the difference in the complete virtual correction is essentially zero at both endpoints $v=0,1$ and reaches a maximum of $3.3 \times 10^{-5}$ units of the Born cross section for $v \approx 0.8$. Most of the difference is found to be due to the mass correction terms. Without these terms, the results would agree to within at most $10^{-5}$, and much less over most of the range. The mass corrections agree in both the soft and hard limits at $1 \mathrm{GeV}$. The soft agreement mirrors that found analytically in the previous section.

Figure 3 examines the NNLL contribution to the JMWY and KR results at $500 \mathrm{GeV}$. Figure 3(a) shows the integrated distributions with the NLL contribution from Eq. (22) subtracted. It is seen that the complete results,

(b) differential

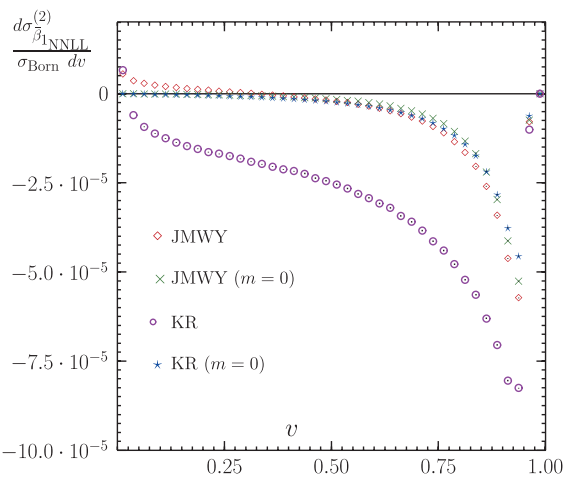

FIG. 2 (color online). The differences between the exact results and the NLL result are shown at a CMS energy of 1 GeV both for (a) a cross section integrated to a cutoff $v_{\max }$ and for (b) a partially differential cross section in $v$. 
(a) integrated

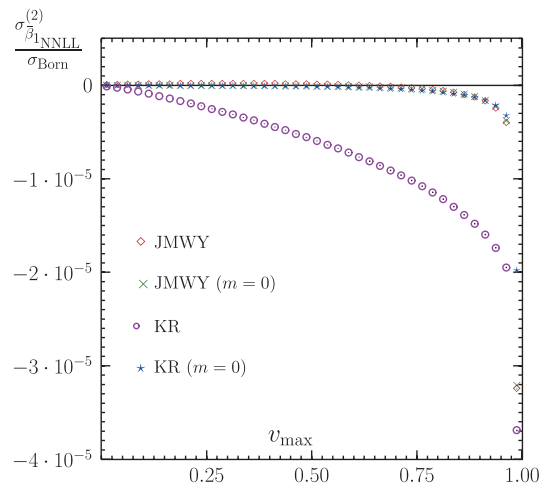

(b) differential

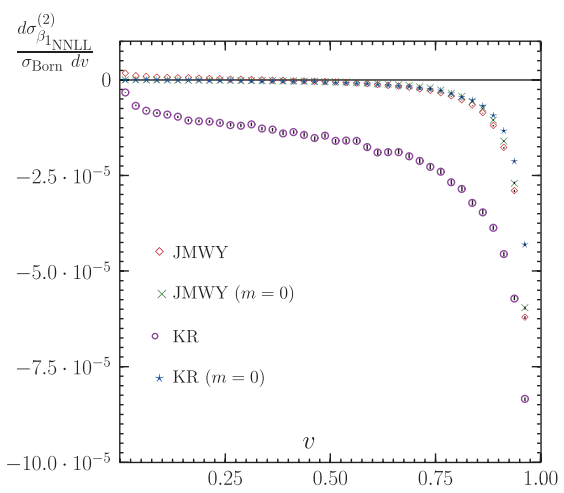

FIG. 3 (color online). The differences between the exact results and the NLL result are shown at a CMS energy of $500 \mathrm{GeV}$ both for (a) a cross section integrated to a cutoff $v_{\max }$ and for (b) a partially differential cross section in $v$.

including mass corrections, differ by at most $1.6 \times 10^{-5}$ units of the Born cross section. Again, the bulk of the difference is seen to be due to the mass corrections. Without these, the results would agree to within $10^{-6}$, except for the last bin. The last bin shows that the integrated contribution of the virtual photon factors over the entire range of photon energies differs by $5 \times 10^{-6}$ units of the Born cross section.

Differential results are compared in Fig. 3(b) up to $v=$ 0.975 . The final data point is omitted to permit the rest of the plot to be shown in better resolution. For the last data point $(v=0.9875)$, the KR result was $7.0 \times 10^{-4}$ for the complete expression and $6.6 \times 10^{-4}$ for the massless part, while the JMWY result was $11.4 \times 10^{-4}$ for the complete expression and $11.3 \times 10^{-4}$ for the massless part. The difference between the differential results at this point is likely to be enhanced due the steepness of the distribution. For the remaining points, the difference is less than $3 \times$ $10^{-5}$. In the limit when $v \rightarrow 1$, additional numerical issues may arise which are separate from the collinear singularities, so it is possible that some of the difference in this limit may be numerical.

The absolute sizes of the NNLL contributions to the integrals in Figs. 2(a) and 3(a) are also of interest, since these determine the range of $v$ for which the much simpler NLL expression can be substituted for the complete result. For the JMWY results at $500 \mathrm{GeV}$, the NNLL contribution does not exceed $4 \times 10^{-6}$ units of the Born cross section for cuts up to $v_{\max }=0.95$, while for larger $v$, the NLL contribution can reach the $3.2 \times 10^{-5}$ level. At $1 \mathrm{GeV}$, the NNLL contribution to the JMWY result does not exceed $7 \times 10^{-6}$ for the full range of photon energies.

The fact that the NNLL contributions to the KR result are typically larger is mostly due to the mass corrections, which are considerably greater in the KR expression over most of the range of $v$. The mass corrections in the JMWY expression reach a level of at most $10^{-6}$ at $1 \mathrm{GeV}$, and are smaller at $500 \mathrm{GeV}$. In contrast, the $\mathrm{KR}$ mass corrections are typically of the order of $2 \times 10^{-5}$ at intermediate photon energies.

While we have expressed the comparisons in units of the nonradiative Born cross section, it is also useful to express them in units of the integrated cross section $\sigma_{1}^{\text {ISR }}$ for single real initial-state radiation, since there is always at least one photon in the radiative return context. Integrating the differential cross section of Ref. [25], including the virtual photon contribution which cancels the IR divergence in the real emission cross section, gives $\sigma_{1}^{\text {ISR }}=0.113 \sigma_{\text {Born }}$ at $\sqrt{s}=1 \mathrm{GeV}$ and $\sigma_{1}^{\mathrm{ISR}}=0.980 \sigma_{\text {Born }}$ at $\sqrt{s}=500 \mathrm{GeV}$. Dividing by $\sigma_{1}^{\text {ISR }}$ instead of $\sigma_{\text {Born }}$ would give essentially the same comparisons at $500 \mathrm{GeV}$, but increase the differences by a factor on the order of 10 at $1 \mathrm{GeV}$.

\section{CONCLUSIONS}

In this paper, we have compared two versions of the exact virtual corrections to initial-state bremsstrahlung in fermion pair production in detail, examining the collinear limits (NLL behavior) analytically both with and without the explicit mass corrections in each case. We found that the results of JMWY and KR agree to NLL order, both with and without masses, and that both results have the same soft limits.

Numerical comparisons of the two virtual photon expressions were made by integrating them with the $\mathcal{K} \mathcal{K}$ MC. The results were presented both in terms of integrated YFS residuals, to match comparisons made earlier, and in differential form, to permit comparison of the virtual photon corrections as a function of photon energy. It was found that the differential results agree to within $3 \times 10^{-5}$ units of the Born cross section across almost all photon energies, for both high energy (500 GeV) and lower energy (1 GeV) scattering. The difference between the integrals of the YFS beta functions over the full photon energy range are also on the order of $10^{-5}$. This level of agreement is compatible with that found in earlier studies at $200 \mathrm{GeV}$ [15-18]. 
The two virtual photon expressions compared agree much better when the mass corrections are omitted from both. This is because the mass corrections in the KR expression are typically of order $10^{-5}$, while the JMWY mass corrections tend to be of order $10^{-6}$ units of the Born cross section over most of the photon energy range. Part of this difference could be due to the structure of the expressions, since the JMWY expression was created with the expectation of using it in the $\mathcal{K} \mathcal{K} \mathrm{MC}$, while the $\mathrm{KR}$ result is foreign to this MC. However, some difference is not surprising, due to the difference in approach to the mass corrections. The JMWY result follows the approach of Berends et al. [19], adding the essential mass corrections for the collinear limits, and KR use an expansion in powers of $m_{e}^{2} / p_{i} \cdot k$. The results are very different analytically, with the KR expressions containing many more terms.

The results found in this paper give a clear estimate of the size of the difference in two exact, mass-corrected matrix elements for initial-state radiation in fermion pair production. We have also found that these matrix elements agree to NLL order, and have compared two approaches to adding mass corrections to the matrix element. Such information is important for estimating the precision of these matrix elements in applications to such processes as the LEP2 final data analysis, radiative return at $\Phi$ and $B$ factories, and anticipated future ILC physics.

\section{ACKNOWLEDGMENTS}

Work partly supported by Department of Energy Grant No. DE-FG02-05ER41399 and NATO Grant No. PST.CLG.980342.

\section{APPENDIX: STABILIZED FUNCTIONS}

The stabilized expressions in this paper depend on two sets of functions introduced to handle cancellations in differences of logarithms and dilogarithms with very similar arguments. The functions $\operatorname{Lf}_{n}(x)$ and $\operatorname{Sf}_{n}(x, y)$ are defined recursively by

$$
\begin{aligned}
\mathrm{Lf}_{0}(x) & =\ln (1+x), \quad \mathrm{Lf}_{n+1}(x)=\frac{1}{x}\left(\mathrm{Lf}_{n}(x)-\mathrm{Lf}_{n}(0)\right), \\
\mathrm{Sf}_{0}(x, y) & =\mathrm{Sp}(x+y), \quad \mathrm{Sf}_{n+1}(x, y)=\frac{1}{y}\left(\mathrm{Sf}_{n}(x, y)-\mathrm{Sf}_{n}(x, 0)\right)
\end{aligned}
$$

with $\operatorname{Sp}(x)$ the Spence dilogarithm function, which is also denoted $\mathrm{Li}_{2}(x)$.

Expansions can be used to evaluate $\operatorname{Lf}_{n}(x)$ for $x$ small or $\operatorname{Sf}_{n}(x, y)$ for $y$ small. For the logarithmic difference function $\mathrm{Lf}_{n}$,

$$
\operatorname{Lf}_{n}(x)=-\sum_{k=n}^{\infty} \frac{(-1)^{k}}{k} x^{k-n}
$$

Differences between the functions $\operatorname{Lf}_{n}(x)$ for similar arguments may be calculated using the identity

$$
\begin{aligned}
\frac{1}{y}\left\{\operatorname{Lf}_{n}(x+y)-\operatorname{Lf}_{n}(x)\right\}= & \frac{1}{(x+y)^{n}}\left\{\frac{1}{1+x} \operatorname{Lf}_{1}\left(\frac{y}{1+x}\right)\right. \\
& \left.-\sum_{k=1}^{n}(x+y)^{k-1} \operatorname{Lf}_{k}(x)\right\}
\end{aligned}
$$

valid for $n \geq 1$. Some identities for arguments containing ratios are

$$
\begin{aligned}
(x+y) \operatorname{Lf}_{1}\left(\frac{y}{x}\right) & =x \operatorname{Lf}_{1}\left(\frac{-y}{x+y}\right), \\
(x+y)^{2} \operatorname{Lf}_{2}\left(\frac{y}{x}\right) & =-x^{2} \operatorname{Lf}_{2}\left(\frac{-y}{x+y}\right)-x(x+y), \\
(x+y)^{3} \operatorname{Lf}_{3}\left(\frac{y}{x}\right) & =x^{3} \operatorname{Lf}_{3}\left(\frac{-y}{x+y}\right)+\frac{1}{2} x y(x+y) .
\end{aligned}
$$

For the dilogarithmic difference function $\mathrm{Sf}_{n}$,

$$
\operatorname{Sf}_{n}(x, y)=\sum_{k=n}^{\infty} \frac{y^{k-n}}{k !} \operatorname{Sp}^{(k)}(x)
$$

where $\operatorname{Sp}^{(k)}(x)$ is the $k$ th derivative of $\operatorname{Sp}(x)$, which may be calculated recursively using

$$
\begin{aligned}
& \operatorname{Sp}^{(1)}(x)=\operatorname{Lf}_{1}(-x) \\
& \operatorname{Sp}^{(n+1)}(x)=\frac{1}{x}\left\{\frac{(n-1) !}{(1-x)^{n}}-n \operatorname{Sp}^{(n)}(x)\right\} \quad \text { for } n \geq 1
\end{aligned}
$$

In the limit where both $x$ and $y$ are small, a double expansion is useful,

$$
\mathrm{Sf}_{n}(x, y)=\sum_{k=n}^{\infty} \sum_{l=n}^{k} \frac{1}{k^{2}}\left(\begin{array}{l}
k \\
l
\end{array}\right) x^{k-l} y^{l-n}
$$

The functions $\operatorname{Lf}_{n}(x)$ have a logarithmic singularity at $x=-1$. The functions $\operatorname{Sf}_{n}(x, y)$ are singular at $x=1$ for $n \geq 1$, but can be calculated in for $x$ approaching 1 with $x+y<1$ using the identity 


$$
\begin{aligned}
\operatorname{Sf}_{n}(x, y)= & (-1)^{n+1} \operatorname{Sf}_{n}(1-x,-y)+\frac{1}{x^{n-1}} \operatorname{Lf}_{1}(-x) \operatorname{Lf}_{n}\left(\frac{y}{x}\right)-\frac{1}{(x-1)^{n-1}} \operatorname{Lf}_{1}(x-1) \operatorname{Lf}_{n}\left(\frac{y}{x-1}\right) \\
& -\frac{y^{q}}{x^{p}(x-1)^{p}} \operatorname{Lf}_{p}\left(\frac{y}{x}\right) \operatorname{Lf}_{p}\left(\frac{y}{x-1}\right)+\sum_{k=1}^{p-1}\left\{\frac{x^{k-n}}{(1-x)^{k}} \operatorname{Lf}_{n-k}\left(\frac{y}{x}\right)+(-1)^{n} \frac{(1-x)^{k-n}}{x^{k}} \operatorname{Lf}_{n-k}\left(\frac{y}{x-1}\right)\right\}
\end{aligned}
$$

where $n \geq 1, p$ is the integer part of $(n+1) / 2$ and $q=1$ for $n$ even, $q=0$ for $n$ odd. A useful special case is

$$
\operatorname{Sf}_{1}(x, y)=\operatorname{Sf}_{1}(1-x,-y)-\operatorname{Lf}_{1}(x-1) \operatorname{Lf}_{1}\left(\frac{y}{x-1}\right)-\frac{1}{x} \ln (1-x-y) \operatorname{Lf}_{1}\left(\frac{y}{x}\right)
$$

[1] F. A. Berends, W. L. Van Neerven, and G. J. H. Burgers, Nucl. Phys. B297, 429 (1988), and references therein.

[2] M. Igarashi and N. Nakazawa, Nucl. Phys. B288, 301 (1987).

[3] S. Jadach, M. Melles, B. F. L. Ward, and S. A. Yost, Phys. Rev. D 65, 073030 (2002).

[4] J. H. Kühn and G. Rodrigo, Eur. Phys. J. C 25, 215 (2002).

[5] S. Binner, J. H. Kühn, and K. Melnikov, Phys. Lett. B 459, 279 (1999).

[6] K. Melnikov, F. Nguyen, B. Valeriani, and G. Venanzoni, Phys. Lett. B 477, 114 (2000).

[7] H. Czyż and J. H. Kühn, Eur. Phys. J. C 18, 497 (2001).

[8] G. Rodrigo, A. Gehrmann-De Ridder, M. Guilesaume, and J. H. Kühn, Eur. Phys. J. C 22, 81 (2001).

[9] G. Rodrigo, H. Czyż, J. H. Kühn, and M. Szopa, Eur. Phys. J. C 24, 71 (2002).

[10] H. Czyż, A. Grzelinska, J. H. Kühn, and G. Rodrigo, Eur. Phys. J. C 33, 333 (2004).

[11] S. Jadach, B. F. L. Ward, and Z. Wass, Phys. Rev. D 63, 113009 (2001); Comput. Phys. Commun. 130, 260 (2000), and references therein.

[12] H. Czyż, A. Grzelinska, J. H. Kühn, and G. Rodrigo, Nucl. Phys. B, Proc. Suppl. 131, 39 (2004).

[13] S. jadach, Acta Phys. Pol. B 36, 2387 (2005).

[14] D. R. Yennie, S. C. Frautschi, and H. Suura, Ann. Phys. (N.Y.) 13, 379 (1961); see also K. T. Mahanthappa, Phys. Rev. 126, 329 (1962), for a related analysis.
[15] C. Glosser, S. Jadach, B. F. L. Ward, and S. Yost, Phys. Lett. B 605, 123 (2005).

[16] S. A. Yost, C. Glosser, S. Jadach, and B. F. L. Ward, hepph/0409041.

[17] S. A. Yost, C. Glosser, S. Jadach, and B. F. L. Ward, in Proceedings of the 32nd International Conference on High Energy Physics (ICHEP 2004), Beijing (World Scientific, Singapore, 2005), p. 478.

[18] S. A. Yost, S. Jadach, and B. F. L. Ward, Acta Phys. Pol. B 36, 2379 (2005).

[19] F. A. Berends, R. Kleiss, P. De Causmaecker, R. Gastmans, W. Troost, and T. T. Wu, Nucl. Phys. B206, 61 (1982).

[20] F.A. Berends, P. De Causmaecker, R. Gastmans, R. Kleiss, W. Troost, and T. T. Wu, Nucl. Phys. B264, 243 (1986).

[21] Z. Xu, D.-H. Zhang, and L. Chang, Nucl. Phys. B291, 392 (1987).

[22] R. Kleiss and W. J. Stirling, Nucl. Phys. B262, 235 (1985); Phys. Lett. B 179, 159 (1986).

[23] J. A. M. Vermaseren, Symbolic Manipulation with FORM (Computer Algebra Netherlands, Amsterdam, 1991).

[24] S. Jadach, M. Melles, B. F. L. Ward, and S. A. Yost, Phys. Lett. B 377, 168 (1996).

[25] F. A. Berends, W. L. van Neerven, and G. J. H. Burgers, Nucl. Phys. B297, 429 (1988). 